\title{
Interaction of $\mathrm{CH}_{4}$ and $\mathrm{H}_{2} \mathrm{O}$ in ice mixtures
}

\author{
Víctor J. Herrero, Óscar Gálvez,* Belén Maté and Rafael Escribano
}

Received 28th October 2009, Accepted 18th January 2010

First published as an Advance Article on the web 18th February 2010

DOI: $10.1039 / \mathbf{b} 922598 f$

Ice mixtures of methane and water are investigated by means of IR spectroscopy in the 14-60 K range. The spectroscopic research is focused on the symmetry-forbidden $\nu_{1}$ band of $\mathrm{CH}_{4}$ and the dangling bond bands of water. The $\nu_{1}$ band is visible in the spectra of the mixtures, revealing a distorted methane structure which co-exists with the normal crystalline methane. The water dangling bond bands are found to increase their intensity and appear at red-shifted frequency when distorted methane is present. Methane adsorbed on water micropores or trapped inside the amorphous solid water structure is assumed to be responsible for these effects. $\mathrm{CH}_{4}$ mobility in water ice depends on the deposition method used to prepare the samples and on the temperature. After warming the samples to $60 \mathrm{~K}$, above the methane sublimation point, a fraction of $\mathrm{CH}_{4}$ is retained in the water ice. An adsorption isotherm analysis is performed yielding the estimation of the desorption energy of $\mathrm{CH}_{4}$ on $\mathrm{H}_{2} \mathrm{O}$ amorphous surfaces.

\section{Introduction}

Icy systems composed of mixtures of frozen species are found in many different environments. On Earth, ice particles are constituents of clouds, where water can have other companions which often play fundamental atmospheric roles, like e.g. sulfuric acid in acid rain episodes, or nitric acid in polar stratospheric clouds. Snow-deposited ice usually contains gaseous species trapped inside, like $\mathrm{CO}_{2}$, which is used to date geological strata in permanent ice regions like Antarctica or Greenland. Other planets and satellites of our Solar system have been shown to hold frozen amounts of different species. ${ }^{1-4}$ The rocky core which constitutes comet nuclei is assumed to be surrounded by an icy layer, where water, $\mathrm{CO}$, $\mathrm{CO}_{2}, \mathrm{CH}_{4}$ and $\mathrm{CH}_{3} \mathrm{OH}$ are the main components. Further beyond, interstellar grains are also believed to be formed by a silicate or carbonaceous core covered by an icy mantle of light species, of which water is again usually the most abundant, hydrogen being assumed to have escaped or been recombined to produce other more stable species.

The $\mathrm{CH}_{4} / \mathrm{H}_{2} \mathrm{O}$ system is particularly interesting, from both astrophysical and geological points of view. It has been detected in comets and in interstellar grain mantles, ${ }^{5-8}$ and the recent results of the Cassini-Huygens mission to Titan suggest the existence of methane clathrates under the surface of the satellite. ${ }^{9-11}$ There is growing interest in a possible methane sequestration into, or release from, $\mathrm{CH}_{4} / \mathrm{H}_{2} \mathrm{O}$ mixtures, and, in general, in all possible kinds of association between these species, including clathrates, aerosols and in the solid state. $^{12-14}$

Infrared spectroscopy is well suited to study these systems. The profile (shape, width, and peak position) of IR bands in the spectra of ice mixtures strongly depends on the structure of the ice and hence IR spectroscopy can reveal structural

Instituto de Estructura de la Materia, CSIC, Serrano 123, 28006 Madrid, Spain information on the sample, often related to its generation method and thermal history. Laboratory experiments conducted on $\mathrm{CH}_{4} / \mathrm{H}_{2} \mathrm{O}$ samples reported changes in peak position, profiles and relative strength of IR bands with temperature and methane concentration. ${ }^{15-20}$ There are also several studies on the morphology of water ices through $\mathrm{CH}_{4}$ adsorption experiments. ${ }^{21-23}$

We present in this article an investigation on methane/water ice mixtures in the $14-60 \mathrm{~K}$ temperature range. The main tool used in this study is transmission IR spectroscopy. Following a description of the experimental method, the paper presents the main spectroscopic results, based on the observation of the dipole-forbidden breathing vibration of methane and the dangling bond bands of water, and the variation of these features with temperature and methane concentration. These spectroscopic findings are then discussed. In the following section, the desorption energy of methane from amorphous solid water (ASW) is estimated from isothermal adsorption experiments. The main conclusions are summarized at the end of the paper.

\section{Experimental}

All experiments were performed in a high vacuum chamber (base pressure in the $10^{-8}$ mbar range) described in detail before. $^{24,25}$ Ice films of $\mathrm{H}_{2} \mathrm{O}, \mathrm{CH}_{4}$ and of mixtures of the two species were vapour-deposited on a cold silicon substrate cooled to $14 \mathrm{~K}$ by an ARS cryostat. The temperature of the substrate was measured with $\mathrm{Si}$ diodes and could be controlled, with $1 \mathrm{~K}$ accuracy, between 14 and $300 \mathrm{~K}$ by means of a Lakeshore temperature control module. $\mathrm{H}_{2} \mathrm{O}$ vapour (distilled water, three times freeze-pump-thawed) and $\mathrm{CH}_{4}(99.95 \%$, Air Liquide) were admitted into the chamber through independent needle valves. The ice films were characterized by normal incident transmission spectroscopy at $2 \mathrm{~cm}^{-1}$ resolution (corresponding to $c a .0 .5 \mathrm{~cm}$ mirror displacement in the interferometer), after co-adding 500 scans 
for each spectrum. The spectra were recorded using a Bruker Vertex70 FTIR spectrometer purged with dry air. The system was provided with a differentially pumped quadrupole mass spectrometer (QMS) that allowed to check the purity of each species before deposition and to control the vapour stoichiometry during the deposition process.

The QMS was calibrated from measurements of the growth rate of solid films of the pure species. ${ }^{26}$ By simulating our transmission spectra with the adequate optical constants taken from the literature, the film thicknesses needed for the calculation of the growth rate were derived. The typical thickness values ranged between 80 and $400 \mathrm{~nm}$. For water ice grown at $14 \mathrm{~K}$, we used the optical constants of Hudgins et $a l .{ }^{15}$ at $10 \mathrm{~K}$. A sticking coefficient of 1 and a density of $0.7 \mathrm{~g} \mathrm{~cm}^{-3}$ were assumed at this temperature. ${ }^{23,27}$ For $\mathrm{CH}_{4}$ at $14 \mathrm{~K}$, a sticking coefficient of $1^{28}$ and a density of $0.47 \mathrm{~g} \mathrm{~cm}^{-3}$ were used. ${ }^{29} \mathrm{~A}$ mean value of the optical constants given by Hudgins et al. ${ }^{15}$ for pure $\mathrm{CH}_{4}$ at 10 and $20 \mathrm{~K}$ was used to simulate the IR spectra, because the $\mathrm{CH}_{4}$ spectrum undergoes considerable changes over this temperature interval. The calibration of the QMS remained stable in the course of the present experiments as verified by periodic checks. The estimated error in the absolute pressure values was $\sim 10 \%$. Deposition pressures ranged between approximately $2 \times 10^{-7}$ and $1 \times 10^{-3}$ mbar.

Films of mixed $\mathrm{CH}_{4} / \mathrm{H}_{2} \mathrm{O}$ ices were prepared by sequential or simultaneous deposition of the vapours. In the first case, water ice was initially formed by depositing water vapour at 14,40 or $50 \mathrm{~K}$ and then $\mathrm{CH}_{4}$ vapour was added at specified temperatures. In the second scheme, $\mathrm{H}_{2} \mathrm{O}$ and $\mathrm{CH}_{4}$ were introduced into the chamber simultaneously, at the appropriate ratio, and allowed to deposit on the substrate, held at 14 or $40 \mathrm{~K}$. To study the thermal behavior of the ices, the temperature was gradually raised at $10 \mathrm{~K} \mathrm{~min}^{-1}$, and spectra were recorded at 30 and $60 \mathrm{~K}$ for ices deposited at $14 \mathrm{~K}$, and at $60 \mathrm{~K}$ for ices deposited at 40 or $50 \mathrm{~K}$.

The integrated values of the infrared absorption bands and the corresponding integrated absorption coefficients, $A$, were used to calculate the column densities of water and $\mathrm{CH}_{4}$ in the ice mixtures via the following relation:

$$
N=\frac{\text { Int }}{A}
$$

where $N$ is the column density of absorbing molecules (the number of absorbers per unit area) in the beam and Int $=\int \ln \left(I_{0} / I\right) \mathrm{d} \nu$ is the integrated band peak area. The molecular ratio $\mathrm{CH}_{4} / \mathrm{H}_{2} \mathrm{O}$ in the mixtures is determined as the quotient of the respective column densities.

For this purpose the bands selected were the water $\mathrm{OH}$-stretching band around $3.1 \mu \mathrm{m}\left(3200 \mathrm{~cm}^{-1}\right)$ due to the symmetric and antisymmetric vibrations, and the $\nu_{4}$ band of $\mathrm{CH}_{4}$ at $\sim 7.7 \mu \mathrm{m}\left(1300 \mathrm{~cm}^{-1}\right)$. The absorption coefficients for these bands were taken from Hudgins et al. ${ }^{15}$ For our samples at low temperatures we used $A=8.085 \times 10^{-18} \mathrm{~cm} /$ molecule for pure $\mathrm{CH}_{4}$ ice (corrected assuming a $\mathrm{CH}_{4}$ density of $0.47 \mathrm{~g} \mathrm{~cm}^{-3}$ ), and $A=2.43 \times 10^{-16} \mathrm{~cm} /$ molecule for water (corrected assuming a $\mathrm{H}_{2} \mathrm{O}$ density of $0.7 \mathrm{~g} \mathrm{~cm}^{-3}$ ). A small temperature correction ${ }^{15}$ was applied in mixtures at 40 and
$60 \mathrm{~K}$ yielding $8.17 \times 10^{-18} \mathrm{~cm} /$ molecule for $\mathrm{CH}_{4}$, and $2.53 \times$ $10^{-16} \mathrm{~cm} /$ molecule for water.

As mentioned above, the film thickness, $d$, of the pure species is derived by simulating our transmission spectra with the adequate optical constants taken from the literature. ${ }^{15}$ The same procedure is used to estimate the thickness of the ASW samples used for sequential deposition. For simultaneously deposited ice mixtures the determination of film thickness is more complicated and only a rough estimate can be given. We have used the following expression:

$$
d=\frac{N_{\mathrm{H}_{2} \mathrm{O}}+N_{\mathrm{CH}_{4}}}{\rho}
$$

where $N_{\mathrm{H}_{2} \mathrm{O}}$ and $N_{\mathrm{CH}_{4}}$ are the column densities of water and methane, respectively, and $\rho$ is the density of the ice mixture. This density of the mixture is unknown, in principle, but it can be conjectured that for vapor deposited ices from a diffuse background gas at $14 \mathrm{~K}$, it will lie between the values of pure $\mathrm{H}_{2} \mathrm{O}$ and pure $\mathrm{CH}_{4}$ deposited under the same conditions. For a given molecular ratio, we have taken the corresponding average density. Note that the range of densities contemplated is not too large (between $\sim 0.5 \mathrm{~g} \mathrm{~cm}^{-3}$ for $\mathrm{CH}_{4}$ and $0.7 \mathrm{~g} \mathrm{~cm}^{-3}$ for $\mathrm{H}_{2} \mathrm{O}$ ). The estimated uncertainties in the $d$ values for mixtures are in the $20-30 \%$ range. It should be stressed at this point that an accurate knowledge of the film thickness is not crucial for the objectives of the present work.

\section{Spectroscopic results and discussion}

Fig. 1 presents an overview of spectra of a $\mathrm{CH}_{4} / \mathrm{H}_{2} \mathrm{O}=0.4$ co-deposited sample at $14 \mathrm{~K}$ and after warming at $60 \mathrm{~K}$. Spectra of the pure components are well known and are not reproduced here. They can be seen for instance in Gálvez et al. ${ }^{19}$ We discuss in this section the most relevant spectral features specific for the mixtures, namely the methane $\nu_{1}$ band and the water dangling bond bands.

\subsection{The $\mathrm{CH}_{4} \nu_{1}$ band}

One of the most striking findings in this investigation is the observation of the $\nu_{1}$ band of $\mathrm{CH}_{4}$, corresponding to the breathing vibration, which is forbidden by symmetry in the

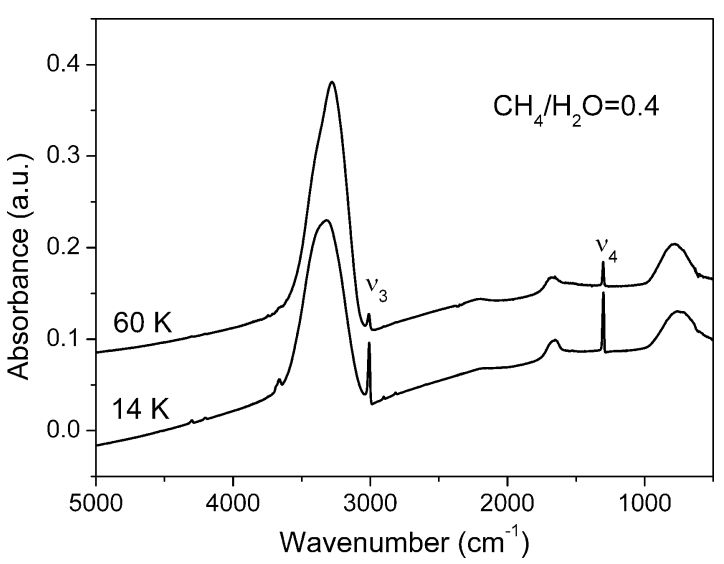

Fig. 1 IR spectrum of a $\mathrm{CH}_{4} / \mathrm{H}_{2} \mathrm{O}=0.4$ co-deposited ice mixture at $14 \mathrm{~K}$ and heated at $60 \mathrm{~K}$. The estimated film thickness is $160( \pm 40) \mathrm{nm}$. Spectra are offset for clarity. 
infrared spectra of gas phase and in pure $\mathrm{CH}_{4}$ crystals. It is present in all our spectra of $\mathrm{CH}_{4} / \mathrm{H}_{2} \mathrm{O}$ mixtures, at $\sim 2904 \mathrm{~cm}^{-1}$, and its spectroscopic behaviour can be used to derive information on the type of $\mathrm{H}_{2} \mathrm{O}-\mathrm{CH}_{4}$ association and interaction. Fig. 2 shows enlarged views of the 2750-2950 $\mathrm{cm}^{-1}$ spectral region of both simultaneously and sequentially deposited samples, illustrating also their temperature variation. The samples were formed by deposition at $14 \mathrm{~K}$ and later warmed up to $60 \mathrm{~K}$. The spectra reveal different behaviour of this band depending on the formation process, which can be better appreciated by comparison to the symmetry-allowed $\nu_{1}+\nu_{4}$ combination band. In simultaneous deposition experiments (upper panel), the $\nu_{1}$ band is weak but noticeable from the beginning of the deposition at $14 \mathrm{~K}$, and its relative intensity with respect to $\nu_{1}+\nu_{4}$ does not change in the warming process until $60 \mathrm{~K}$, where most of the $\mathrm{CH}_{4}$ is already evaporated. At this temperature some methane fraction must remain as the strongest $\mathrm{CH}_{4}$ vibrations, and traces of $\nu_{1}$, are still seen. On the other hand, in sequential deposition experiments, the $\nu_{1}$ band is extremely weak initially, becomes stronger at $30 \mathrm{~K}$, always with respect to the combination band, and then again a trace only is left at $60 \mathrm{~K}$. A possible explanation of these facts is proposed below, after the results on the dangling bonds are presented.

\subsection{The $\mathrm{H}_{2} \mathrm{O}$ dangling bond bands}

Dangling bonds in solid water give rise to two bands (DB1 and DB2, at 3720 and $3696 \mathrm{~cm}^{-1}$ respectively) attributed to $\mathrm{OH}$ vibrations of doubly- or triply-coordinated surface molecules, and have been related to the porosity of the sample (see e.g. ref. 23 and 30). Their intensity relative to the fundamental vibrational bands of water rises with increasing surface to

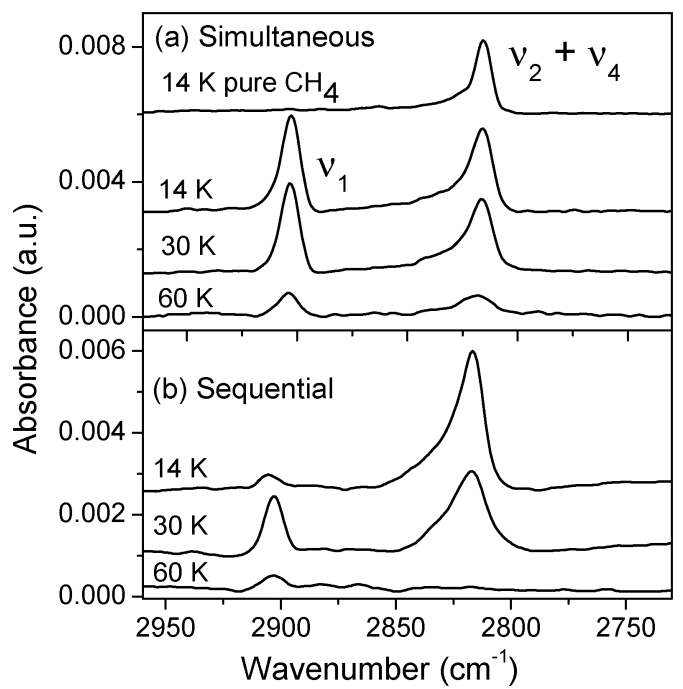

Fig. 2 IR spectra in the $2750-2950 \mathrm{~cm}^{-1}$ region. (a) $\mathrm{CH}_{4} / \mathrm{H}_{2} \mathrm{O}=0.4$ simultaneously deposited ice mixture at $14 \mathrm{~K}$ and warmed afterwards to the indicated temperatures; the spectrum of a pure methane ice is also included for comparison. (b) $\mathrm{CH}_{4} / \mathrm{H}_{2} \mathrm{O}$ sequential sample generated at $14 \mathrm{~K}$ and warmed to 30 and $60 \mathrm{~K}$. Spectra are offset for clarity. A film thickness of $180( \pm 40) \mathrm{nm}$ has been estimated for the simultaneously deposited sample in panel (a) and of $330( \pm 30) \mathrm{nm}$ for the sequential sample of panel (b). The pure methane ice film is $80( \pm 10)$ nm thick.

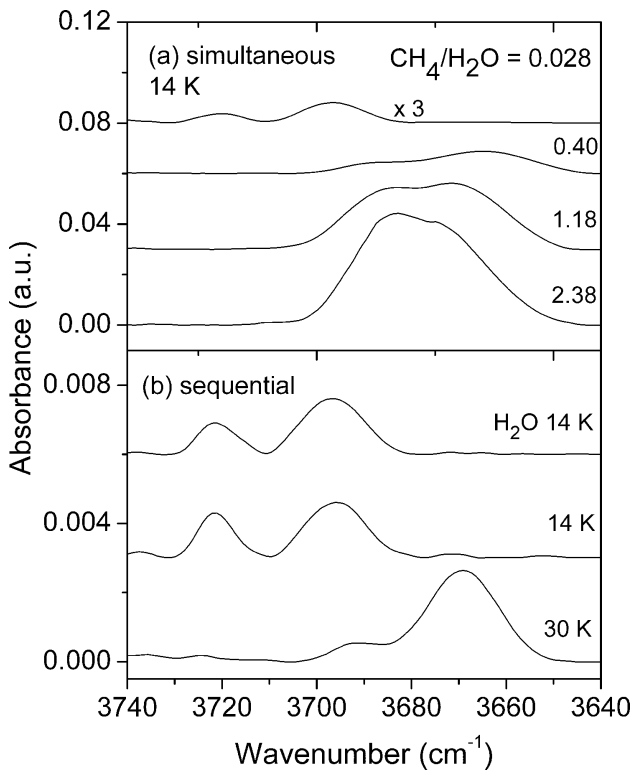

Fig. 3 Detail of the dangling bonds spectral IR region. (a) $\mathrm{CH}_{4} / \mathrm{H}_{2} \mathrm{O}$ simultaneously deposited samples generated at $14 \mathrm{~K}$ at varying molecular abundance ratios in the solid. The estimated film thicknesses are $360,180,330$ and $460 \mathrm{~nm}$, from top to bottom with uncertainties in the $20-30 \%$ range. (b) Sequential sample generated at $14 \mathrm{~K}$ and heated to $30 \mathrm{~K}$. A pure water spectrum is also presented for comparison (upper trace). Both samples are $250 \mathrm{~nm}$ thick with $10 \%$ uncertainty.

volume proportion in the ice. Our experiments have detected spectral variations for these bands in $\mathrm{CH}_{4} / \mathrm{H}_{2} \mathrm{O}$ mixtures, and again different behaviour is observed associated to the kind of deposition scheme. Fig. 3 presents spectra in the $3640-3740 \mathrm{~cm}^{-1}$ region. The upper panel presents spectra of simultaneously deposited samples with roughly the same amount of water molecules, except for the top spectrum which has twice the water content for a better appreciation of the weak DB signals. When the sample is formed by co-deposition of the vapours at $14 \mathrm{~K}$ (upper panel), the DB1 and DB2 bands are not altered for very low $\mathrm{CH}_{4}$ proportion, but when the methane concentration is increased, the bands are red-shifted and their intensity grows markedly. In sequentially deposited mixtures, the DB1 and DB2 bands are not altered by the admission of methane at $14 \mathrm{~K}$. However, when the sample is warmed at $30 \mathrm{~K}$, a red-shift of these bands is observed, as in the co-deposition experiment.

\subsection{Discussion}

In the following interpretation of the spectroscopic results we will make implicit use of information gathered from the adsorption isotherm analysis presented below. The main conclusion of such analysis concerns the microporous structure of AWS ices at $14 \mathrm{~K}$.

The $\nu_{1}$ band of methane is a strong feature in Raman spectra, either of the gas phase ${ }^{31}$ or from noble gas matrices, ${ }^{32}$ and has recently been reported in infrared spectra of $\mathrm{CH}_{4}$ ice mixtures by Hodyss et al. ${ }^{20}$ and Gálvez et al. ${ }^{19}$ The observation of this breathing vibration in infrared spectra implies a breakdown of the symmetry of the system, and consequently the formation of a distorted form of $\mathrm{CH}_{4}$. The adsorption of 
$\mathrm{CH}_{4}$ molecules on water ice surfaces, or the presence of very small methane crystallites distorted from their cubic structure through interactions with the amorphous water environment, could cause such an effect. This result bears some similarity to the observation of a distorted form of $\mathrm{CO}_{2}$ in ice mixtures with water ${ }^{25,33,34}$ or methanol. ${ }^{35}$ In the $\mathrm{CO}_{2}$ case, the distorted $\mathrm{CO}_{2}$ structure gave rise to band shifts of several wavenumbers with respect to the bands of pure or undistorted $\mathrm{CO}_{2}$, which was also present in the mixtures. For methane the interaction with water seems weaker, and $\mathrm{CH}_{4}$-dist, as we may call this distorted structure, is mainly manifested by the appearance of the forbidden $\nu_{1}$ band. Small shifts or broadenings are also observed on some of the other bands, ${ }^{19}$ but they do not allow an independent and simultaneous identification of both methane structures.

To achieve a better understanding of this problem, we performed series of measurements varying the $\mathrm{CH}_{4} / \mathrm{H}_{2} \mathrm{O}$ concentration ratio and also the sample temperature. Fig. 4 summarizes some of our results for mixtures formed in simultaneous deposition experiments. The upper panel shows the increase in the absolute intensity of the "forbidden" $\nu_{1}$ band of $\mathrm{CH}_{4}$ with increasing methane concentration, for mixtures co-deposited at 14 and $40 \mathrm{~K}$. The next graph presents an interesting magnitude, the measured intensity ratio between this $\nu_{1}$ band and the strong fundamental $\mathrm{CH}_{4} \nu_{4}$ band. The forbidden $\nu_{1}$ band is due to $\mathrm{CH}_{4}$-dist only, whereas $\nu_{4}$ contains contributions from both distorted and normal methane, and therefore this quotient gives some idea of the relative amount of $\mathrm{CH}_{4}$-dist in the sample. The graph shows that this ratio grows very quickly at $14 \mathrm{~K}$ for small values of the $\mathrm{CH}_{4} / \mathrm{H}_{2} \mathrm{O}$ concentration, reaches a maximum at $\mathrm{CH}_{4} / \mathrm{H}_{2} \mathrm{O}$ ratios of about 0.4 and then drops. The data measured at $40 \mathrm{~K}$ indicate a parallel drop in the distorted to normal methane ratio, occurring at lower methane to water concentrations. In the pure $\mathrm{CH}_{4}$ limit, with no interaction with water to cause the distortion, $\mathrm{CH}_{4}$-dist should not exist. On the other hand, the intensity of DB bands, displayed in the lower graph of Fig. 4, indicates that these bands become stronger with increasing $\mathrm{CH}_{4}$ concentration. Our interpretation of these phenomena is the following. When methane and water are admitted simultaneously into the chamber, they mix intimately providing distorted environments that favour the appearance of $\mathrm{CH}_{4}$-dist. When methane is added in higher concentrations, it tends to form larger "islands" where the $\mathrm{CH}_{4}$-dist relative concentration is smaller, but where the surface in contact with amorphous water ice, responsible for the DBs, is also higher.

Fig. 4 depicts also the same magnitudes after heating the samples to $60 \mathrm{~K}$. At that temperature, most of the methane has sublimated, but a small, constant fraction remains. This fraction probably corresponds basically to $\mathrm{CH}_{4}$-dist in the inner recesses of the bulk ASW, keeping the same value that it had for the lowest $\mathrm{CH}_{4} / \mathrm{H}_{2} \mathrm{O}$ proportions. In a recent work, ${ }^{19}$ the amount of methane retained in co-deposited samples heated at $60 \mathrm{~K}$ was estimated to have a saturation value of $\sim 10 \pm 2 \%$ irrespective of the initial $\mathrm{CH}_{4}$ excess concentration. In the former investigations on $\mathrm{CO}_{2} / \mathrm{H}_{2} \mathrm{O}$ and $\mathrm{CO}_{2} / \mathrm{CH}_{3} \mathrm{OH}$ mixtures mentioned above, ${ }^{25,33-35}$ some $\mathrm{CO}_{2}$ was also retained above its sublimation temperature, $\sim 90 \mathrm{~K}$.

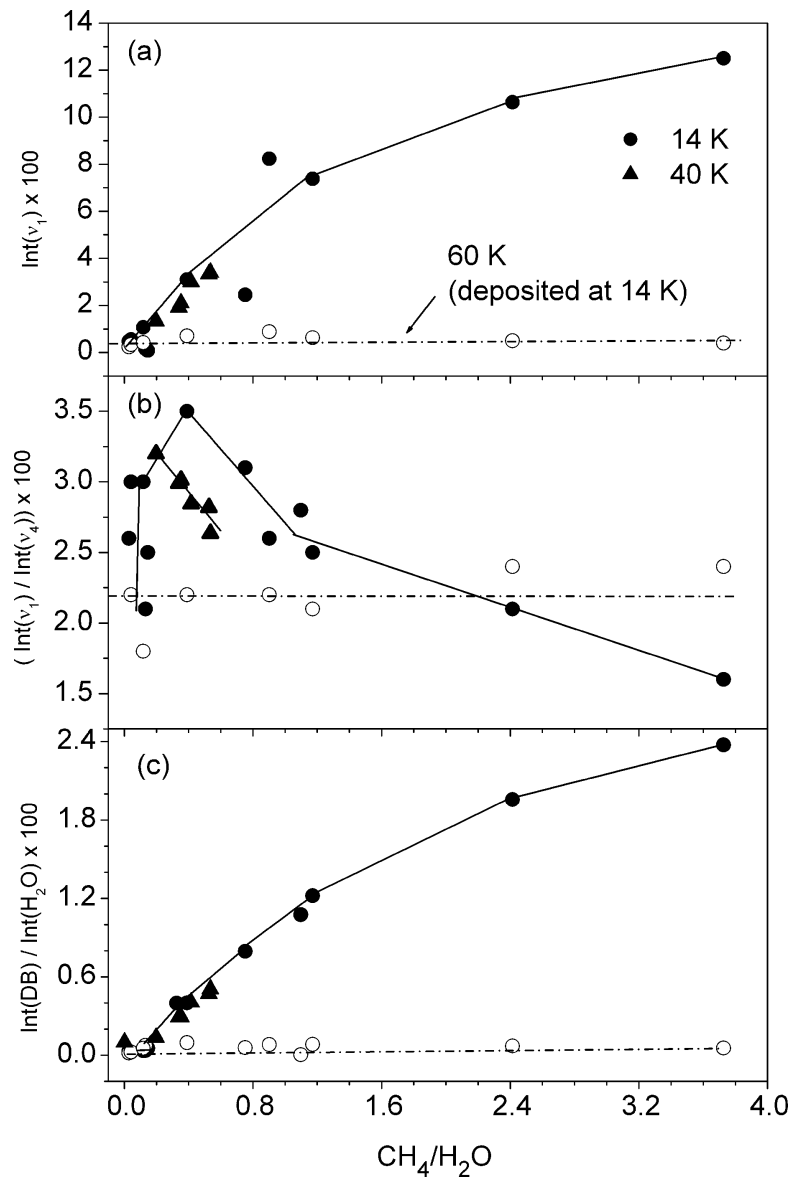

Fig. 4 (a) Integrated intensity of the "forbidden" $\nu_{1}$ band of methane versus $\mathrm{CH}_{4} / \mathrm{H}_{2} \mathrm{O}$ molecular ratio for co-deposited samples. Solid circles: ice films generated at $14 \mathrm{~K}$; triangles: deposition at $40 \mathrm{~K}$; open circles: samples deposited at $14 \mathrm{~K}$ and heated to $60 \mathrm{~K}$. (b) Methane $\nu_{1} / \nu_{4}$ integrated band intensity ratio from the same experiments. (c) Intensity ratio of $\mathrm{DB}$ bands with respect to the $\mathrm{OH}$ stretching band of water from the same experiments. All symbols as in previous panels.

When ASW is formed first, addition of methane at $14 \mathrm{~K}$ results in the growth of a $\mathrm{CH}_{4}$ layer adsorbed on the external surface of the ice, with a minimum interaction with water, characterized by a very weak $\nu_{1}$ band of $\mathrm{CH}_{4}$-dist, and no change in the DB spectral bands as compared to water ice. However, if the sample is heated to $30 \mathrm{~K}$, some fraction of methane molecules enter the ice and become adsorbed in narrow channels or micropores, either due to a higher $\mathrm{CH}_{4}$ diffusion rate at that temperature or because of changes taking place in the structure of ASW. This part of the methane concentration is in the $\mathrm{CH}_{4}$-dist form, with a visible $\nu_{1}$ band. Further heating to $60 \mathrm{~K}$ induces sublimation of most $\mathrm{CH}_{4}$, but, as for co-deposited samples, a small amount is retained, showing a weak but non-null $\nu_{1}$ band and no trace of $\nu_{2}+\nu_{4}$. The results from both experimental methods confirm the existence of a distorted form of $\mathrm{CH}_{4}$, kept in narrow and internal cavities of ASW, where it can stay above the sublimation temperature of methane. Note that the micropore size currently estimated for this type of ASW samples does not exceed three $\mathrm{CH}_{4}$ molecular diameters, ${ }^{23}$ which implies a very close interaction between $\mathrm{CH}_{4}$ and the surrounding $\mathrm{H}_{2} \mathrm{O}$ environment. 
Fig. 5 presents the results of a study on the dependence of these effects on the thickness of the ASW formed prior to $\mathrm{CH}_{4}$ addition. The figure represents the $\mathrm{CH}_{4} / \mathrm{H}_{2} \mathrm{O}$ intensity ratio for the $\mathrm{CH}_{4} \nu_{1}$ band (solid circles) and the $\mathrm{CH}_{4} \nu_{4}$ band (open circles), for ices formed at $14 \mathrm{~K}$ and warmed to 30 and $60 \mathrm{~K}$, respectively. The $\mathrm{CH}_{4} \nu_{4}$ band is chosen at the higher temperature because $\nu_{1}$ is too weak to allow intensity measurements to be performed. Both intensity ratios decrease with growing thickness of the ASW layer, indicating that the $\mathrm{CH}_{4}$ introduced into the ice must occupy internal positions close to the surface, and is not evenly distributed into the bulk, which would yield constant growth with the ice thickness. Raut et al. ${ }^{23}$ reported similar results for $\mathrm{CH}_{4}$ adsorption experiments carried out at $40 \mathrm{~K}$. Making use of the integrated intensity data mentioned above, it is possible to estimate from the values represented in Fig. 5 the relative amount of distorted methane retained in sequentially deposited samples. This value depends on the water ice film thickness. At $60 \mathrm{~K}$, it varies from $1.6 \%$ for the thicker ASW films studied (400 nm) to $2.3 \%$ for the thinner ones $(80 \mathrm{~nm})$. We believe that in these thin films we are close to the limit of total diffusion of methane. In consequence, approximately four times more methane is retained at $60 \mathrm{~K}$ when the sample is generated by simultaneous deposition than in sequential experiments. This result is in accordance with intuitive expectations that the trapping of methane in the bulk of the ice or in inner cavities should be more efficient in co-deposited samples.

\section{Adsorption isotherms}

Information about the morphology of the ASW samples and about the desorption energy of $\mathrm{CH}_{4}$ from the ice surface can be derived from adsorption isotherms. The upper panel of Fig. 6 shows the results for adsorption of $\mathrm{CH}_{4}$ on ASW at 40 and $50 \mathrm{~K}$. The two amorphous ice samples had the same thickness $(\sim 195 \mathrm{~nm})$. In the figure, the molecular ratio of adsorbed methane to ASW, given by the respective integrated band intensities, is represented as a function of the equilibrium methane pressure normalized by the corresponding saturation

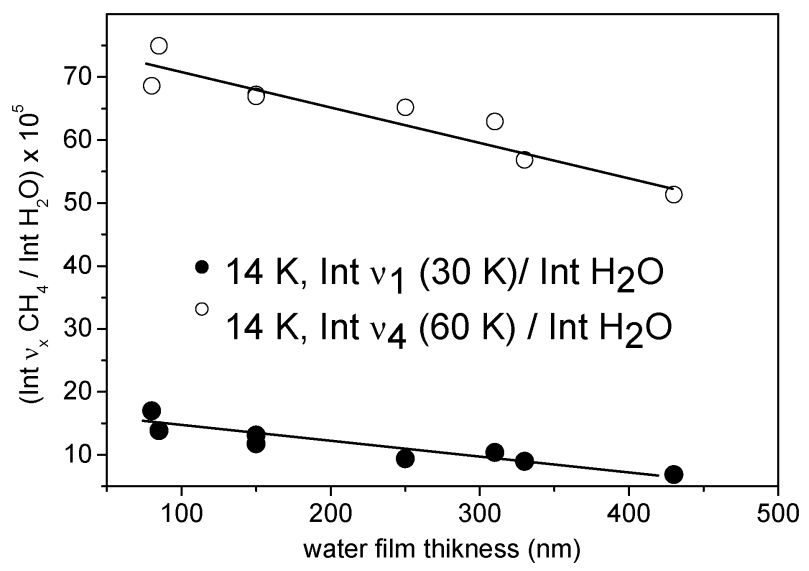

Fig. 5 Solid circles: integrated $\nu_{1} \mathrm{CH}_{4} / \mathrm{OH}$ stretching $\mathrm{H}_{2} \mathrm{O}$ band intensity ratio versus water ice film thickness for ices generated at $14 \mathrm{~K}$ and heated to $30 \mathrm{~K}$. Open circles: integrated $\nu_{4} \mathrm{CH}_{4} / \mathrm{OH}$ stretching $\mathrm{H}_{2} \mathrm{O}$ band intensity ratio versus water ice film thickness for the same samples heated to $60 \mathrm{~K}$.
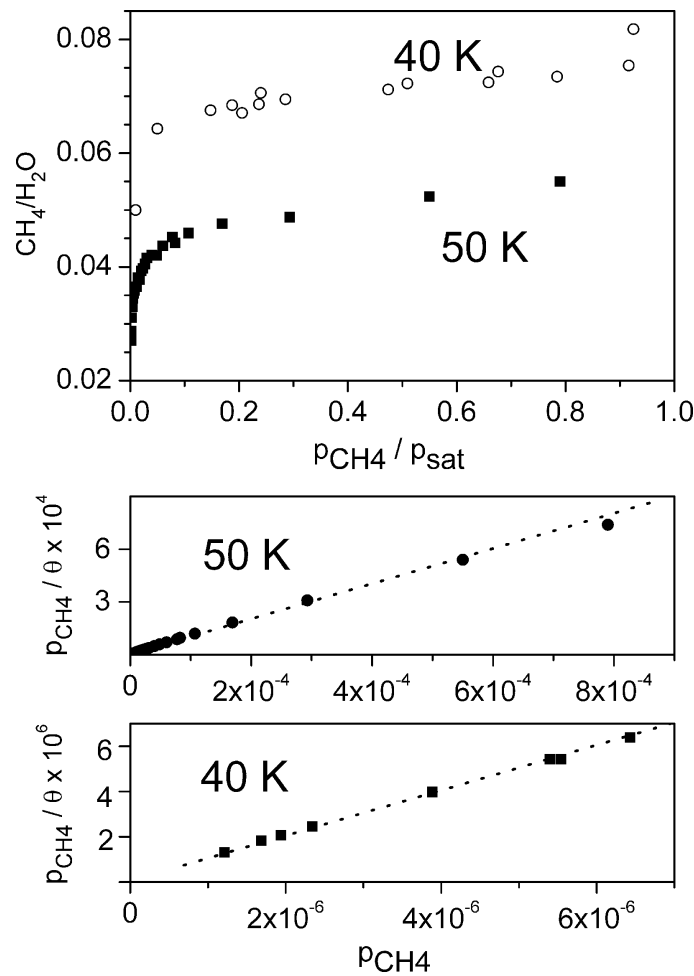

Fig. 6 Upper panel: $\mathrm{CH}_{4} / \mathrm{H}_{2} \mathrm{O}$ molecular ratio for sequential ice mixtures generated at $40 \mathrm{~K}$ and $50 \mathrm{~K}$, versus the relative methane pressure ratio used for its generation. The water ice film thickness was $195 \mathrm{~nm}$ for both temperatures. The saturation pressures were $8.2 \times$ $10^{-6}$ mbar and $1.0 \times 10^{-3}$ mbar for 40 and $50 \mathrm{~K}$ respectively. Lower panels: fit of the data from the upper panel to Langmuir isotherms (eqn (5) in the text).

pressure. The resulting isotherms are of type $\mathrm{I},{ }^{36,37}$ with a roughly flat plateau characteristic of microporous solids as expected for ASW at this temperature (see ref. 38 and references therein). The amount of adsorbed methane decreases with growing temperature, indicating that the ASW becomes less porous when it is formed at higher temperatures. Horimoto and coworkers ${ }^{22}$ studied methane absorption on $\mathrm{D}_{2} \mathrm{O}$ ices grown at $25 \mathrm{~K}$ and annealed at different temperatures. They conclude that up to $80 \mathrm{~K}$ the microporous structure of ices generated at $25 \mathrm{~K}$ is not affected by the heating. Our observations show that the temperature of generation of the ASW samples affects the microporous structure more drastically than the annealing process.

The data of Fig. 6 can be fitted to type I Langmuir isotherms:

$$
\theta=\frac{x}{x_{m}}=\frac{b p}{1+b p}
$$

where $\theta$ is the surface coverage, i.e. the fraction of a monolayer actually occupied by adsorbed molecules, and $b$ is the adsorption coefficient given by:

$$
b=K \exp \left(E_{\mathrm{d}} / R T\right)
$$

where $K$ is a constant and $E_{\mathrm{d}}$ the desorption energy. Eqn (3) can be expressed as:

$$
\frac{p}{\theta}=\frac{1}{b}+p=C+p
$$


In the two lower panels of Fig. 6, the measured data have been represented as $(p / \theta) v s$. $p$. The surface coverage, $\theta$, for each $\mathrm{CH}_{4} / \mathrm{H}_{2} \mathrm{O}$ value has been estimated by assuming that unit coverage is attained at $p_{\text {sat }}$. A fit of the data to straight lines with unit slope (eqn (5)) allows the determination of the adsorption coefficient, which is the inverse of the intercept, $C$. Combining the data from 40 and $50 \mathrm{~K}$ and using eqn (4) a desorption energy $E_{\mathrm{d}}=0.074 \pm 0.010 \mathrm{eV}$ is obtained. The use of the Langmuir isotherm for the derivation of $E_{\mathrm{d}}$ assumes tacitly that the adsorption energy is independent of site or surface coverage. A more elaborate treatment based on the isotherms of Freundlich ${ }^{37}$ and Dubinin-Astakhov ${ }^{39}$ for microporous solids was employed by Raut et al. ${ }^{23}$ for the analysis of their data on the $40 \mathrm{~K}$ adsorption of $\mathrm{CH}_{4}$ deposited on ASW from collimated vapour sources at various incidence angles. The most probable binding energies for methane adsorption reported by these authors range from 0.083 to $0.077 \mathrm{eV}$ depending on the incidence angle. These values are in good agreement with the desorption energy determined in the present work.

In their studies about the adsorption of different gases on ices of astrophysical interest, Sandford and Allamandola ${ }^{40}$ used the "sticking zero" condition of the gas on the surface for the estimate of desorption energies. This method, which has been used in previous works of our group, ${ }^{33,35}$ assumes that deposition ceases when the average residence time of the molecules on the surface is equal to the time during which the ice surface is hit by a number of molecules equivalent to one monolayer coverage, $t_{\mathrm{m}}$. For a given ice temperature, the "sticking zero" condition describes the equilibrium situation between the gas and the ASW at the limit of $p_{\text {sat. The }}$ desorption energy is estimated with the expression: ${ }^{28,33}$

$$
E_{\mathrm{d}}=R T \ln \left(\nu_{0} t_{\mathrm{m}}\right)
$$

where $\nu_{0}$ is the frequency of oscillation of the molecules on the surface and $t_{\mathrm{m}}$ is given by.

$$
t_{\mathrm{m}}=n_{\mathrm{s}} /(1 / 4) n\langle v\rangle
$$

where $n_{\mathrm{s}}$ is the monolayer surface density; $n$, the molecular density for a given pressure and $\langle v\rangle$ the average molecular velocity at the corresponding gas temperature. To apply this procedure, the effective mean $\mathrm{CH}_{4}$ molecular area and the frequency of oscillation of the adsorbed $\mathrm{CH}_{4}$ molecules on the surface, $\nu_{0}$, are required. We have taken a molecular area of $14.77 \AA^{2}$ from the $\mathrm{CH}_{4}$ ice density given by Satorre et al. ${ }^{29}$ and $\nu_{0}=1.57 \times 10^{12} \mathrm{~s}^{-1}$ for $\mathrm{CH}_{4}$ adsorbed on $\mathrm{Cu}(001)$ at $58 \mathrm{~K}$ as measured by Graham et al. ${ }^{41}$ The energy values calculated with eqn (6) are 0.093 and $0.095 \mathrm{eV}$ for data at 40 and $50 \mathrm{~K}$, respectively. With these data we can give an estimate of the desorption energy, $E_{\mathrm{d}}=0.094 \pm 0.010 \mathrm{eV}$, which provides an average desorption energy for the different adsorption sites available in the ASW. This result is somewhat higher than the $E_{\mathrm{d}}=0.074 \pm 0.01$ determined from the Langmuir isotherm and also than the binding energies reported by Raut and coworkers. $^{23}$

It should be mentioned here that the collision frequency from gas kinetic theory used for the derivation of eqn (6) might not be accurate for micropores of very small diameter; still this procedure provides a reasonable estimate of $E_{\mathrm{d}}$. The energy of desorption of $\mathrm{CH}_{4}$ molecules from water-ice is about three times lower that the corresponding value for $\mathrm{CO}_{2}{ }^{28,33}$

\section{Summary and conclusions}

This paper presents the results of an investigation on $\mathrm{CH}_{4} / \mathrm{H}_{2} \mathrm{O}$ ice mixtures at cold temperatures by means of infrared spectroscopy, aiming to improve the present understanding of the interactions in these species. The main conclusions drawn from this work are summarized below.

Our samples were prepared by vapour deposition at temperatures of 14,40 and $50 \mathrm{~K}$, with $\mathrm{CH}_{4} / \mathrm{H}_{2} \mathrm{O}$ ratios ranging from 0.028 to 3.6. The deposited ice films were also heated up to $60 \mathrm{~K}$ to study their temperature evolution.

The appearance of the symmetry forbidden $\nu_{1}$ band of methane in the solid samples is indicative of the presence of $\mathrm{CH}_{4}$ molecules in a distorted environment within the ice. By comparison to similar but more marked effects encountered in $\mathrm{CO}_{2} / \mathrm{H}_{2} \mathrm{O}$ and $\mathrm{CO}_{2} / \mathrm{CH}_{3} \mathrm{OH}$ mixtures, we conclude that the distortions of the molecular environment must be caused by weak interactions.

Vapour deposited amorphous water ices in the 40-50 K temperature interval are microporous as demonstrated by the type $\mathrm{I}$ isotherms obtained for the adsorption of $\mathrm{CH}_{4}$. The application of simple adsorption models leads to desorption energies in the $\sim 0.074-0.094 \mathrm{eV}$ range. These results are in agreement with recent literature estimates. The effective area for adsorption decreases appreciably when the deposition temperature grows from 40 to $50 \mathrm{~K}$. This effect of deposition temperature is in contrast with that of annealing, which has recently been reported not to change the microporous structure of the sample over the temperature range considered. ${ }^{22}$

A joint analysis of the evolution of the water dangling bond bands and the $\nu_{1}$ methane band shows that at $14 \mathrm{~K}$ methane deposited on amorphous water ice does not enter the microporous structure. At $30 \mathrm{~K}$ the methane molecules have enough mobility to diffuse inside the pores. ASW may have experienced a rearrangement of its structure that also facilities the methane mobility. The micropores, with an estimated size of less than three $\mathrm{CH}_{4}$ molecular diameters, could provide the asymmetrical environment of close water-methane interaction responsible for the appearance of the forbidden $\nu_{1}$ band. In contrast, when methane and water are admitted simultaneously, distorted methane is formed from the lowest temperature, $14 \mathrm{~K}$. Islands of solid $\mathrm{CH}_{4}$ seem to form in the ice for $\mathrm{CH}_{4} / \mathrm{H}_{2} \mathrm{O}$ molecular ratios larger than 0.3 .

Heating the ice samples to $60 \mathrm{~K}$ leads to evaporation of most of the methane, but a small fraction is retained, largely in the $\mathrm{CH}_{4}$ distorted structure. This fraction is about four times larger in co-deposited mixtures than in the sequentially deposited samples.

\section{Acknowledgements}

This research has been carried out with funding from the Spanish Ministry of Education, Project FIS2007-61686. O.G. acknowledges financial support from CSIC, "JAE-Doc" program. 


\section{References}

1 M. H. Moore, R. L. Hudson and R. F. Ferrante, Earth, Moon, Planets, 2003, 92, 291.

2 R. Hodyss, J. D. Goguen, P. V. Johnson, C. Campbell and I. Kanik, Icarus, 2008, 197, 152.

3 D. A. Williams, W. A. Brown, S. D. Price, J. M. C. Rawlings and S. Viti, Astronomy \& Geophys., 2007, 48, 25.

4 G. Notesco, D. Laufer and A. Bar-Nun, Icarus, 1997, 125, 471.

5 A. C. A. Boogert, W. A. Schutte, A. G. G. M. Tielens, D. C. B. Whittet, F. P. Helmich, P. Ehrenfreund, P. R. Wesselius and T. de Graauw, Astron. Astrophys., 1996, 315, L377.

6 J. Licandro, N. Pinilla-Alonso, M. Pedani, E. Oliva, G. P. Tozzi and W. M. Grundy, Astron. Astrophys., 2006, 445, L35.

7 K. I. Öberg, A. C. A. Boogert, K. M. Pontoppidan, G. A. Blake, N. J. Evans, F. Lahuis and E. F. van Dishoeck, Astrophys. J., 2008, 678, 1032.

8 G. Zasowski, F. Kemper, D. M. Watson, E. Furlan, C. J. Bohac, C. Hull and J. D. Green, Astrophys. J., 2009, 694, 459.

9 T. C. Owen, H. Niemann, S. Atreya and M. Y. Zolotov, Faraday Discuss., 2006, 133, 387.

10 H. B. Niemann and the GCMS Team, Nature, 2005, 438, 779.

11 M. Choukroun, G. Tobie and O. Grasset, Lunar \& Planet. Science XXXVII, 2006, 1640.

12 R. Signorell and M. Jetzki, Phys. Rev. Lett., 2007, 98, 013401.

13 E. Dartois and D. Deboffle, Astron. Astrophys., 2008, 490, L19.

14 C. C. Wang, P. Zielke, O. F. Sigurbjörnsson, C. R. Viteri and R. Signorell, J. Phys. Chem. A, 2009, 113(42), 11129.

15 D. M. Hudgins, S. A. Sandford, L. J. Allamandola and A. G. G. M. Tielens, Astrophys. J. Suppl., 1993, 86, 713.

16 A. C. A. Boogert, W. A. Schutte, F. P. Helmich, A. G. G. M. Tielens and D. H. Wooden, Astron. Astrophys., 1997, 317, 929.

17 O. Kerkhof, W. A. Schutte and P. Ehrenfreund, Astron. Astrophys., 1999, 346, 990.

18 M. P. Bernstein, D. P. Cruikshank and S. A. Sandford, Icarus, 2006, 181, 302.

19 O. Gálvez, B. Maté, V. J. Herrero and R. Escribano, Astrophys. J., 2009, 703, 2101.

20 R. Hodyss, P. V. Johnson, J. V. Stern, J. D. Goguen and I. Kanik, Icarus, 2009, 200, 338.

21 L. Chaix and F. Dominé, J. Phys. Chem. B, 1997, 101, 6105.
22 N. Horimoto, H. S. Kato and M. Kawai, J. Chem. Phys., 2002, 116(11), 4375.

23 U. Raut, M. Famá, B. D. Teolis and R. A. Baragiola, J. Chem. Phys., 2007, 127, 204713.

24 E. Carrasco, J. M. Castillo, R. Escribano, V. J. Herrero, M. A. Moreno and J. Rodríguez, Rev. Sci. Instrum., 2002, 73, 3469.

25 O. Gálvez, B. Maté, V. J. Herrero and R. Escribano, Icarus, 2008, 197, 599.

26 B. Maté, A. Medialdea, M. A. Moreno, R. Escribano and V. J. Herrero, J. Phys. Chem. B, 2003, 107(40), 11098.

27 Z. Dohnálek, G. A. Kimmel, P. Ayotte, R. S. Smith and B. D. Kay, J. Chem. Phys., 2003, 118, 364.

28 S. A. Sandford and L. J. Allamandola, Astrophys. J., 1990, 355, 357.

29 M. A. Satorre, M. Domingo, C. Millán, R. Luna, R. Vilaplana and C. Santonja, Planet. Space Sci., 2008, 56(13), 1748.

30 V. Buch and J. P. Devlin, J. Chem. Phys., 1991, 94, 4091.

31 D. Bermejo, R. Escribano and J. M. Orza, J. Mol. Spectrosc., 1977, 65, 345 .

32 M. Sagara and M. Ikezawa, J. Phys. Soc. Jpn., 1983, 52, 327.

33 O. Gálvez, I. K. Ortega, B. Maté, M. A. Moreno, B. MartínLlorente, V. J. Herrero, R. Escribano and P. J. Gutiérrez, Astron. Astrophys., 2007, 472(2), 691.

34 B. Maté, O. Gálvez, B. Martín-Llorente, M. A. Moreno, V. J. Herrero, R. Escribano and E. Artacho, J. Phys. Chem. A, 2008, 112(3), 457.

35 B. Maté, O. Gálvez, V. J. Herrero and R. Escribano, Astrophys. J., 2009, 690, 486.

36 F. Rouquerol, J. Rouquerol and K. Sign, Adsorption by Powders \& Porous Solids, Academic Press, London, 1999.

37 W. Rudzinski and D. H. Everett, Adsorption of Gases on Heterogeneous Surfaces, Academic Press, 1992.

38 R. A. Baragiola, Microporous Amorphous Water Ice Thin Films Properties and Their Astronomical Implications, in Water In Confined Geometries, ed. J. P. Devlin and V. Buch, Springer, Berlin, 2003.

39 M. M. Dubinin and V. A. Astakhov, Adv. Chem., 1971, 102, 69.

40 S. A. Sandford and L. J. Allamandola, Icarus, 1990, 87, 188.

41 A. P. Graham, M. F. Bertino, F. Hofmann, W. Silvestri and J. P. Toennies, J. Chem. Phys., 1997, 106(6), 2502. 